\title{
Dosimetria externa em minas de caulim na região do Seridó do Nordeste do Brasil
}

\section{(External dosimetry in kaolin mines in the Seridó region of Northeast Brazil)}

\author{
I. C. G. Spacov", A. N. C. da Silva**, R. dos S. Amaral, J. A. dos Santos Jr., J. D. Bezerra, \\ E. E. N. de Araújo, M. C. A. Costa \\ Grupo de Radioecologia, Programa de Pós-Graduação em Tecnologias Energéticas e Nucleares, \\ Departamento de Energia Nuclear, Universidade Federal de Pernambuco, \\ Av. Prof. Luiz Freire 1000, Recife, PE, Brasil 50740-540 \\ *isabelspacov@gmail.com,**ary_casado@yahoo.com.br
}

\begin{abstract}
Resumo
A atividade mineradora pode apresentar potenciais riscos radiológicos em decorrência da presença de radionuclídeos primordiais associados aos minérios explorados. No nordeste brasileiro, as mineradoras de caulim estão estabelecidas principalmente na região da Província Pegmatítica Borborema-Seridó localizada entre os estados da Paraíba e do Rio Grande do Norte. O minério da caulinita traz agregado materiais formados por elementos das séries do U-238 e Th-232, além do K-40. A presença desses radioisótopos naturais pode conferir elevados níveis de radiação ionizante associados ao material extraído das minas. A UNSCEAR divulga a dose efetiva média mundial de exposição às fontes naturais de radiação como $2,42 \mathrm{mSv} \cdot \mathrm{a}^{-1}$. Estudos nacionais e internacionais demonstraram que a atividade radioativa do caulim pode exceder os valores médios mundiais. Este estudo objetivou o mapeamento radiométrico das áreas de exploração do caulim na região do Seridó devido à proximidade com jazidas de urânio. Foi utilizado um detector cintilador de $\mathrm{NaI}(\mathrm{Tl})$ para realizar as medições de taxa de dose efetiva nas proximidades de quatro minas da região e foram obtidos valores que variaram entre 1,37 a 2,94 $\mathrm{mSv} \cdot \mathrm{a}^{-1}$. Os resultados obtidos sugerem a necessidade de uma abordagem mais profunda como a obtenção de amostras de solo e rocha para outras análises.
\end{abstract}

Palavras-chave: radiação ionizante; mapeamento radiométrico, radionuclídeos naturais.

\begin{abstract}
The mining activity may have potential radiological hazards due to the presence of primordial radionuclides associated with the exploited ore. In northeastern of Brazil, kaolin mining companies are mainly located in the region of Borborema-Seridó Pegmatitic Province located between the states of Paraíba and Rio Grande do Norte. The kaolinite ore brings aggregate materials formed by elements belonging to the series of $U-238$ and Th-232, beyond $K-40$. The presence of these natural radioisotopes can confer high levels of ionizing radiation associated with the material extracted from the mines. The UNSCEAR reports the global average effective dose of exposure to natural sources radiation as $2.42 \mathrm{mSv} \cdot \mathrm{y}^{-1}$. National and international studies have shown that the activity of kaolin may exceed the global averages. This study aimed the radiometric mapping of kaolin exploration areas in the Seridó region due to its proximity to uranium deposits. A scintillator detector of $\mathrm{NaI}(\mathrm{Tl})$ was used to perform effective dose rate measurements in the vicinity of four mines in the region, and values that ranged from 1.37 to $2.94 \mathrm{mSv} \cdot \mathrm{y}^{-1}$ were obtained. The results suggest a need for a deeper approach such as obtaining soil and rock samples for more analyzes.
\end{abstract}

Keywords: ionizing radiation, radiometric mapping, natural radionuclides.

\section{INTRODUÇÃ̃O}

Estudos relacionados à radiação ionizante natural de fundo são classificados como de grande importância científica, pois essa classe de radiação representa a maior fonte da exposição para o ser humano [1]. Apesar de todos os estudos realizados, ainda existem muitas dúvidas sobre os efeitos biológicos das baixas doses de radiação. As altas doses produzem efeitos prejudiciais bem definidos sobre o ser humano, porém ocorrem apenas em situações de exposição extremas acidentais, normalmente relacionadas aos radionuclídeos artificiais. Existem muitas lacunas em relação aos riscos à exposição de baixa dose, que ocorre em situações não relacionadas ao trabalho ou a acidentes e pertinentes aos radionuclídeos naturais [2]. O conceito mais básico de dose de radiação ionizante está relacionado à quantidade de energia depositada em qualquer unidade de massa de tecido ou órgão e é definida como dose absorvida. Esta é uma grandeza física, difícil de ser mensurável e representada no Sistema Internacional (SI) por uma unidade denominada Gray. Diferentes tipos de radiação geram diferentes tipos de efeitos biológicos e, por esse motivo, 
a dose absorvida é corrigida por um fator de ponderação que é definido de acordo com o tipo de radiação. Essa dose corrigida é definida como dose equivalente e tem unidade no SI de sievert. Porém, a mesma dose equivalente pode afetar de forma diferente os diversos órgãos ou tecidos. Dessa forma, fatores de ponderação para órgãos e tecidos são utilizados para o cálculo da dose efetiva representada também em sievert. Os estudos atuais utilizam o conceito de taxa de dose efetiva que é definida como a soma ponderada de doses equivalentes recebidas pelo corpo afetado ou exposto, dividida por uma unidade de tempo [3,4]. O Comitê Científico das Nações Unidas para os Efeitos da Radiação Nuclear (UNSCEAR) tem a função de avaliar e relatar os níveis e efeitos da exposição à radiação nuclear artificial ou natural. A UNSCEAR utiliza o valor de $2,4 \mathrm{mSv} \cdot \mathrm{a}^{-1}$ como parâmetro de comparação para dose radioativa natural efetiva proveniente da radiação de fundo para exposição de qualquer grupo de indivíduo. Esse valor continua sendo divulgado, a princípio, como parâmetro de estimação de risco para exposição à baixa dose não sendo causador de dano biológico. Além disso, estudos estatísticos estabeleceram como lognormal a distribuição das taxas de dose ao redor do mundo e um intervalo de 1 até $13 \mathrm{mSv}^{-1} \mathrm{a}^{-1}$ como o valor médio anual esperado em qualquer parte da superfície do planeta. Em diversos locais do Brasil e de outros países, a exposição aferida através de pesquisas forneceu doses cinco vezes maiores [4].

O setor de exploração mineral brasileiro tem grande importância socioeconômica para o país, respondendo por $4,2 \%$ do PIB e $20 \%$ das exportações [5, 6]. O caulim, um tipo de argila de cor branca ou levemente creme e inodora, é formado por silicatos hidratados de alumínio, principalmente a caulinita $\left(\mathrm{Al}_{2} \mathrm{O}_{3} \cdot 2 \mathrm{SiO}_{2} \cdot 2 \mathrm{H}_{2} \mathrm{O}\right)$. Esse mineral surge muitas vezes acompanhado de outros, como mica, quartzo e turmalina. É inorgânico, insolúvel em água, imune aos microrganismos e às mudanças bruscas de temperatura. Entre as diversas aplicações industriais do caulim estão incluídas: produção de cerâmicas, cargas para tintas, borrachas, plásticos e cobertura para papel [7]. Em 2013, a produção mundial de caulim foi da ordem de 37 milhões de toneladas, enquanto que a produção brasileira chegou a 2,14 milhões de toneladas, correspondendo a aproximadamente $5,8 \%$ da produção mundial. Uzbequistão (19,0\%), Estados Unidos $(16,0 \%)$, Alemanha $(12,1 \%)$ e República Checa $(9,0 \%)$ são os maiores produtores de caulim no mundo. A produção brasileira é mais importante na Região Norte, principalmente no estado do Pará que detém $71 \%$ da exploração e beneficiamento, seguido pelo estado do Amapá com aproximadamente $13 \%$. No mercado interno brasileiro as duas principais aplicações são na fabricação de cimento e cerâmica branca $[7,8]$. A produção de caulim é relevante para o Brasil e para a região da Província Pegmatítica Borborema/Seridó (PPBS), localizada no Sertão do Nordeste do Brasil, entre os estados da Paraíba (PB) e do Rio Grande do Norte (RN). Identificada por seu potencial econômico minerador, a área, com cerca de duas dezenas de municípios, ocupando aproximadamente $6000 \mathrm{~km}^{2}$, é muito dependente do próprio caulim, do feldspato, da scheelita e do granito. A lavra do caulim nessa região ocorre há muito tempo, normalmente de maneira rudimentar e predatória, sem análises prévias das jazidas e da geologia formadora dos depósitos [9]. Algumas minas ocorrem a céu aberto e outras em áreas subterrâneas. A exploração normalmente ocorre de forma não mecanizada ou semimecanizada, com riscos de acidentes como desmoronamentos e consequentes soterramentos de trabalhadores. O caulim bruto passa por um processo de beneficiamento para atender as especificações do mercado consumidor, gerando um grande volume de rejeitos sólidos sem valor comercial. Estes são então depositados ao acaso em regiões próximas para evitar o custo do transporte, gerando montanhas de material ao ar livre [7, 10].

Todos os minerais apresentam radioisótopos de origem natural, porém o nível de radiação provenientes desses radionuclídeos é considerado como radiação natural de fundo, não sendo levado em consideração em termos de riscos à saúde e proteção radiológica. Entretanto, as concentrações dos radioisótopos naturais se tornam elevadas, acima de valores considerados seguros, quando ocorrem atividades mineradoras. Qualquer mineral natural referenciado como material radioativo em termos de proteção radiológica é definido como material radioativo de ocorrência natural (NORM) [11], sendo tema de grande interesse para as pesquisas em radioecologia. $\mathrm{O}$ número de trabalhadores envolvidos em atividades mineradoras de caulim na PPBS oscila anualmente de acordo com a economia da região [12]. Esses mineradores estão sujeitos aos riscos operacionais e aos impactos ambientais decorrentes da extração e beneficiamento dos diversos minerais produzidos, porém existe também a possibilidade de exposição interna e externa à radiação ionizante de baixa dose provocada pelo NORM agregado ao minério principal e aos rejeitos. A exposição desses trabalhadores às radiações depende de uma série de fatores, incluindo o tipo de mina, as características geológicas e as condições de trabalho. Um dos fatores agravantes da radiação ionizante natural é que ela expõe o indivíduo por um período prolongado de tempo, a uma taxa relativamente constante [11]. A radiação ionizante natural de fundo pode atingir seres humanos através de diversas vias de transferência (atmosfera, rochas, solos, plantas, animais, águas superficiais e subterrâneas e decompositores) dos diversos ecossistemas [11, 12]. Os mineradores de caulim da região estudada podem sofrer exposição externa devido à emanação dos raios gama provenientes do solo e das rochas pegmatíticas que apresentam um enriquecimento natural dos radioisótopos U-238, Th-232 e de seus descendentes, além do K-40 [13]. Além disso, é possível ocorrer exposição interna devido à inalação e ingestão de partículas atmosféricas provenientes da dispersão da poeira dos rejeitos dispostos próximos às áreas mineradoras [6-9].

Análises químicas do caulim, realizadas durante o processo de beneficiamento, identificaram alto teor de $\mathrm{SiO}_{2}$, e entrevistas realizadas com mineradores desse produto na PPBS constataram problemas de falta de ar em 16,7\% dos entrevistados, além de 83,3\% afirmarem desconhecer 
o que seja silicose, um tipo de fibrose pulmonar crônica e irreversível causada pela inalação da poeira decorrente da extração do caulim, podendo ser evitada através da utilização de máscaras; 66,7\% dos trabalhadores entrevistados declararam a não utilização de equipamentos de proteção individual (EPI). Apesar de não existirem registros ou dados estatísticos relacionados à morbidade hospitalar na região $[11,12]$, os estudos sobre doenças pulmonares ocupacionais demonstram a gravidade da mineração e beneficiamento do caulim [13]. Sabe-se que o caulim utilizado em cerâmicas brancas, na decoração de paredes e pisos, pode conter sais de radioisótopos devido à presença dos radionuclídeos das séries naturais do U-238 e do Th-232, além do K-40 [9, 1416]. Estudos na Índia indicam que a atividade específica radiológica do caulim, utilizado na fabricação de cerâmicas, excede os valores médios mundiais, variando entre 13,6 e 113,5 Bq.kg-1 para o Ra-226 e de 30,5 a 169,4 Bq.kg-1 para o Th-232. A atividade média na crostaé de $32 \mathrm{~Bq} \cdot \mathrm{kg}^{-1}$ para o Ra226 e 45 Bq. $\mathrm{kg}^{-1}$ para o Th-232 [17]. No Egito, 50 amostras de caulim, produzidas no próprio Egito e também importadas do sudeste asiático e da Turquia, foram analisadas através do método de espectroscopia de raios gama e resultaram em concentrações de atividades do Th-232, U-238 e K-40 significativamente altas quando comparadas aos valores médios mundiais na crosta. Valores surpreendentes foram encontrados para o rádio equivalente que variou de 187,8 a $10185,19 \mathrm{~Bq} \cdot \mathrm{kg}^{-1}$. Os autores destacaram ainda uma concentração de atividade na faixa de 0,23 a $8,5 \mathrm{~Bq} \cdot \mathrm{kg}^{-1}$ de Cs-137, radionuclídeo de origem artificial produzido no processo de queima do combustível de usinas nucleares [18]. Porém, pesquisadores turcos realizaram análise para determinar as concentrações de atividades de radionuclídeos naturais em amostras de matéria-prima, como argila, caulim, quartzo, feldspato, dolomita, bauxita e zircônio, e também de produtos acabados, como cerâmicas. O resultado dessa pesquisa na Turquia demonstrou o improvável surgimento de uma exposição radiológica significante para os habitantes das construções produzidas com esses materiais [19]. A conclusão, através dos resultados desses estudos, é que mesmo elevados níveis de radiação natural associada aos radionuclídeos primordiais contidos em materiais de construção podem não submeter os residentes das construções fabricadas com esses materiais a uma exposição radiológica externa significante. Porém, para mineradores e populações adjacentes às minas é fundamental continuar com a realização de estudos mais aprofundados, principalmente devido ao desconhecimento sobre os efeitos da exposição continuada a esses radionuclídeos naturais [18-20].

O Grupo de Radioecologia (RAE) do Departamento de Energia Nuclear (DEN) da Universidade Federal de Pernambuco (UFPE) estuda a emissão radioativa dos radionuclídeos naturais em diversas regiões do Nordeste do Brasil. Artigos, dissertações e teses já foram publicados e atualmente existe um grande interesse na região da PPBS. O estado da Paraíba, no município de São José de Espinharas, já tem registrado uma importante jazida de urânio e terras raras, mapeada em diversos períodos entre os anos 1970 a
1980 pelas empresas Nuclan-Nuclebrás Mineração, Geosol e Crusander do Brasil Mineração. A jazida de urânio é considerada a quinta maior do país com uma reserva total de 10000 toneladas de $\mathrm{U}_{3} \mathrm{O}_{8}$ [21]. Levando em consideração os resultados de trabalhos locais, nacionais e internacionais, foi desenvolvido um projeto de pesquisa com o objetivo de analisar a dose efetiva equivalente em diversos pontos no interior e próximos às minas de caulim da região PPBS. O objetivo principal pretendeu obter o máximo de medições possíveis nos locais de mineração, sendo ao ar livre ou em áreas subterrâneas, nas proximidades e também em áreas de despejos de rejeitos.

\section{MATERIAIS E MÉTODOS}

Inicialmente foi realizada uma pesquisa para identificar as lavras ativas e autorizadas pelo Departamento Nacional de Produção Mineral (DNPM), órgão do Ministério de Minas e Energia responsável por regular a exploração mineral no solo brasileiro. O DNPM possui um sistema acessível pela rede internet (https://sistemas.dnpm.gov.br/SCM/Extra/site/ admin/pesquisarProcessos.aspx) permitindo o acesso às informações das lavras ativas de uma determinada região. Foram pesquisadas no sistema todas as lavras de caulim, com autorização de 01/01/2013 a 30/08/2016, na região PPBS nos estados da Paraíba e Rio Grande do Norte. O objetivo foi identificar as lavras, seus proprietários, a localização, entrar em contato e obter autorização para fazer medições da taxa de dose efetiva equivalente no interior das minas e nas suas cercanias. Uma equipe composta por cinco pesquisadores deslocou-se por um trajeto pré-definido em um automóvel identificado com as logomarcas do Serviço Público Federal, da UFPE e do Grupo RAE. O projeto inicial foi fazer as medições após instalar uma base na cidade de São José de Espinharas no estado da Paraíba, distante $400 \mathrm{~km}$ do ponto de partida que foi a cidade de Recife, capital do estado de Pernambuco. A área de estudo foi delimitada pelos paralelos $6^{\circ} 56^{\prime}$ a $7^{\circ} 03^{\prime}$ de latitude Sul e pelos meridianos $36^{\circ} 41^{\prime}$ a $36^{\circ}$

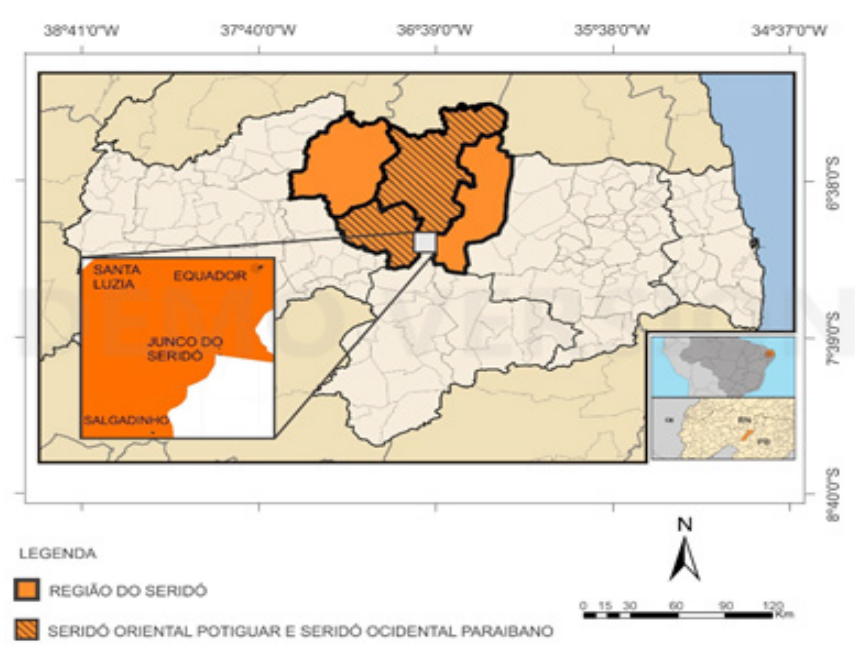

Figura 1: Área de estudo de minas de caulim na PPBS.

[Figure 1: Area of study of kaolin mines in PPBS.] 
45' de longitude Oeste. A partir de São José de Espinharas, o grupo percorreu os seguintes municípios: Junco do Seridó-PB, Equador-RN, Salgadinho-PB, Santa Luzia-PB, Carnaúba dos Dantas-RN, São João do Sabugi-RN e São Mamede-PB, além de povoados e áreas rurais adjacentes. No total, mais de $500 \mathrm{~km}$ foram percorridos, em um período de três dias, numa região com bioma característico de caatinga, temperatura média acima dos $35^{\circ} \mathrm{C}$ e com áreas sem sinalização oficial (Fig. 1).

$\mathrm{O}$ equipamento utilizado para fazer as medições in situ da dose efetiva equivalente foi um detector cintilador discriminador portátil com cristal de iodeto de sódio (NaI), ativado com tálio $(\mathrm{Tl})$, considerado o mais apropriado para detecção de taxas de dose de radiação gama em aferições ambientais [22]. O detector da GF Instruments, Gamma Surveyor, com 512 canais e capaz de registrar uma taxa de contagem de 250000 pulso. $\mathrm{s}^{-1}$, foi operado na faixa de energia entre $100 \mathrm{keV}$ e $3 \mathrm{MeV}$. O aparelho foi composto por uma unidade de controle, com dimensões de 25,6 x 9,0 x $6,0 \mathrm{~cm}$ e massa de $0,5 \mathrm{~kg}$, acoplado a uma sonda gama com dimensões de 9,0 × 12,0 × 29,0 cm, altura de punho de $18,0 \mathrm{~cm}$ e massa de $1,6 \mathrm{~kg}$. A unidade de controle do sistema dispõe de uma capacidade de memória máxima de 32 Mbit para armazenamento dos dados, pode ser interligada a um computador por cabo USB, dispõe de uma bateria recarregável de $12 \mathrm{~V}$ e é de fácil manuseio e manutenção. Um sistema de posicionamento global (GPS) para identificação das coordenadas geodésicas foi acoplado ao detector, permitindo a localização exata de cada ponto verificado. Todo o sistema portátil foi utilizado para fazer as medições no ar, a $1 \mathrm{~m}$ de altura da superfície do solo, com um tempo de contagem de $190 \mathrm{~s}$, definido de acordo com os dados da literatura nacional e internacional para radiação de fundo [22]. O equipamento foi calibrado de acordo com a equação:

$$
\dot{\mathrm{H}}_{\mathrm{E}}=(1,92 \cdot \dot{\mathrm{D}}-1430,87) \cdot 6,14 \cdot 10^{-3}
$$

O detector faz medições em triplicatas dos valores de taxa de dose absorvida $\left(\right.$ nGy.h $\left.^{-1}\right)$ no ar (D́ na Eq. A) e calcula a média aritmética dessa grandeza. A equação de calibração permite o cálculo da taxa de dose efetiva equivalente $\left(\dot{\mathrm{H}}_{\mathrm{E}}\right.$ na Eq. A) no meio ambiente na unidade de $\mathrm{mSv} \cdot \mathrm{a}^{-1}$.

No projeto inicial estava planejada a utilização apenas dos dados obtidos diretamente na área de estudo para a análise estatística dos resultados e correspondentes conclusões. Para a análise estatística, foi programada a utilização do aplicativo gratuito e de código aberto R, v. 3.2.2, facilmente obtido no sítio da internet (https://cran.r-project.org/), além do ambiente de desenvolvimento integrado, grátis e de código aberto RStudio Desktop, v. 1.0.44, que pode ser obtido no endereço da rede internet: https://www.rstudio.com/ products/rstudio/download/. O conjunto amostral deveria ser analisado, em termos de representatividade populacional e adequação à distribuição normal, através do método de Shapiro-Wilk. Devido aos problemas de autorização para acesso às minas apresentados no item de resultados desse trabalho, o número de dados obtidos ficou bastante reduzido para a análise estatística, logo os valores experimentais precisaram ser replicados e tratados estatisticamente para verificar um padrão de normalidade dos dados amostrais através de outro método. Utilizou-se a simulação Monte Carlo - Bootstrap para obtenção de 100000 reamostras das medições obtidas, através do aplicativo estatístico $\mathrm{R}$ [23]. A radiação ionizante proveniente do solo, dos rejeitos e de qualquer outra fonte natural pertencente ao bioma estudado não pode ser analisada de forma apenas pontual. Para um estudo radiométrico mais refinado é necessário ter uma distribuição contínua das taxas de dose equivalentes efetivas que podem atingir os indivíduos da região estudada. Por esse motivo, é preciso calcular as linhas que passam por pontos físicos do terreno nos quais os valores de dose são sempre iguais [24]. Essas curvas são denominadas pelos radioecologistas de cartas de isodoses ou curvas de isodoses. Neste trabalho foi utilizado o aplicativo ArcGIS 9.3 para a construção das curvas de isodoses na região das medições, inserindo no programa as coordenadas geodésicas dos pontos físicos observados e o conjunto de valores obtidos das medições realizadas na região de estudo.

\section{RESULTADOS E DISCUSSÃO}

Através do sistema de consulta de lavras do DNPM foi possível identificar do ano de 2013 até o mês de agosto de 2016, na região PPBS nos estados da Paraíba e Rio Grande do Norte, as minas de caulim ativas e autorizadas a funcionar pelo poder público (Tabela I). Diversas outras cidades possuem mineração de caulim autorizada e em funcionamento, porém a região de interesse envolve principalmente esses municípios localizados na área já identificada como importante jazida de minério de urânio, podendo influenciar na transferência de NORM para o caulim e seus rejeitos. Apesar da identificação positiva das minas e dos contatos prévios mantidos com os proprietários das áreas exploradas, recebendo inclusive autorizações escritas para a entrada nas áreas, no momento do acesso às lavras os responsáveis não autorizaram a entrada do grupo de pesquisadores. As explicações para a negativa de acesso foram: falta de comunicação entre o responsável na mina e o proprietário no momento da execução da pesquisa e a questão da segurança da mina. Foi necessário modificar a metodologia do projeto de pesquisa durante a execução do trabalho de campo para evitar a perda de todo o projeto.

Tabela I - Número de minas de caulim identificadas na região PPBS.

[Table I - Number of kaolin mines identified in the PPBS region.]

\begin{tabular}{ccc}
\hline Município & Estado & Número de minas \\
\hline Equador & $\mathrm{RN}$ & 4 \\
Junco do Seridó & $\mathrm{PB}$ & 12 \\
Salgadinho & $\mathrm{PB}$ & 8 \\
\hline
\end{tabular}


Tabela II - Taxa de dose efetiva equivalente nas proximidades das minas A, B, C e D de caulim. [Table II - Effective equivalent dose rate in the vicinity of kaolin mines A, B, C and D.]

\begin{tabular}{|c|c|c|c|c|c|}
\hline \multirow{2}{*}{ Ponto } & \multirow{2}{*}{ Município-estado } & \multirow{2}{*}{ Mina } & \multicolumn{2}{|c|}{ Coordenadas geodésicas } & \multirow{2}{*}{$\begin{array}{c}\dot{\mathrm{H}}_{\mathrm{E}} \pm \mathrm{dp} \\
\left(\mathrm{mSv} \cdot \mathrm{a}^{-1}\right)\end{array}$} \\
\hline & & & $S$ & WGr & \\
\hline 1 & \multirow{3}{*}{ Equador-RN } & \multirow{3}{*}{ A } & $06^{\circ} 56^{\prime} 39,8^{\prime \prime}$ & $36^{\circ} 41^{\prime} 54,2^{\prime \prime}$ & $1,54 \pm 0,02$ \\
\hline 2 & & & $06^{\circ} 56^{\prime} 39,4^{\prime \prime}$ & $36^{\circ} 41^{\prime} 53,8^{\prime \prime}$ & $1,37 \pm 0,02$ \\
\hline 3 & & & $06^{\circ} 56^{\prime} 38,9^{\prime \prime}$ & $36^{\circ} 41^{\prime} 54,7^{\prime \prime}$ & $1,50 \pm 0,02$ \\
\hline 4 & \multirow{4}{*}{ Equador-RN } & \multirow{4}{*}{ B } & $06^{\circ} 56^{\prime} 40,3^{\prime \prime}$ & $36^{\circ} 41^{\prime} 55,0^{\prime \prime}$ & $1,55 \pm 0,02$ \\
\hline 5 & & & $06^{\circ} 56^{\prime} 40,2^{\prime \prime}$ & $36^{\circ} 41^{\prime} 55,5^{\prime \prime}$ & $1,49 \pm 0,02$ \\
\hline 6 & & & $06^{\circ} 56^{\prime} 42,3^{\prime \prime}$ & $36^{\circ} 41^{\prime} 58,5^{\prime \prime}$ & $1,65 \pm 0,02$ \\
\hline 7 & & & $06^{\circ} 56^{\prime} 42,9^{\prime \prime}$ & $36^{\circ} 41^{\prime} 58,3^{\prime \prime}$ & $1,54 \pm 0,02$ \\
\hline 8 & \multirow{3}{*}{ Junco do Seridó-PB } & \multirow{3}{*}{$\mathrm{C}$} & $06^{\circ} 56^{\prime} 38,7^{\prime \prime}$ & $36^{\circ} 41^{\prime} 52,0^{\prime \prime}$ & $2,29 \pm 0,03$ \\
\hline 9 & & & $06^{\circ} 59^{\prime} 58,3^{\prime \prime}$ & $36^{\circ} 43^{\prime} 23,3^{\prime \prime}$ & $1,64 \pm 0,02$ \\
\hline 10 & & & $07^{\circ} 00^{\prime} 13,0^{\prime \prime}$ & $36^{\circ} 43^{\prime} 22,0^{\prime \prime}$ & $2,01 \pm 0,02$ \\
\hline 11 & \multirow{3}{*}{ Salgadinho-PB } & \multirow{3}{*}{$\mathrm{D}$} & $07^{\circ} 03^{\prime} 23,4^{\prime \prime}$ & $36^{\circ} 45^{\prime} 44,5^{\prime \prime}$ & $1,78 \pm 0,02$ \\
\hline 12 & & & $07^{\circ} 03^{\prime} 22,7^{\prime \prime}$ & $36^{\circ} 45^{\prime} 46,1^{\prime \prime}$ & $1,73 \pm 0,04$ \\
\hline 13 & & & $07^{\circ} 03^{\prime} 21,5^{\prime \prime}$ & $36^{\circ} 45^{\prime} 48,9^{\prime \prime}$ & $2,94 \pm 0,04$ \\
\hline
\end{tabular}

Os pesquisadores identificaram duas minas localizadas nos municípios de Equador-RN com as letras A e B, uma mina identificada com a letra $\mathrm{C}$ no município de Junco do SeridóPB e uma mina localizada no município de SalgadinhoPB identificada como lavra D, nas quais foi possível fazer medições em áreas próximas às lavras, porém não pertencentes aos empreendimentos. Na região da mina A foram feitas três medições, na região da mina B foram feitas quatro medições, na mina $\mathrm{C}$ foram feitas três medições e também na mina $\mathrm{D}$ foram três medições (Tabela II).

Foram realizadas no total apenas 13 medidas em 4 minas diferentes, exclusivamente nas cercanias ou vizinhanças das propriedades próximas ao terreno das lavras, enquanto o projeto inicial previa a abordagem em 24 minas diferentes e o máximo de medições possíveis no interior e na periferia das minas. Como consequência, o número de valores obtidos não foi suficiente para a inferência estatística da situação radioecológica das lavras e os respectivos impactos sobre o ser humano. Foi utilizado então o método de reamostragem não paramétrico Monte Carlo Bootstrap, com reposição dos dados originais, no sentido de obter pseudoamostras independentes que serviram para compor um conjunto de dados válidos para os resultados e conclusões do trabalho. A média aritmética foi a estatística inicialmente utilizada para a formação das reamostras e, em seguida, foi calculado o intervalo de confiança Monte Carlo Bootstrap com o objetivo de verificar se as médias estavam dentro do intervalo médio da população bootstrap [23]. Para cada uma das minas, os valores amostrais reais foram replicados 100000 vezes, através de uma simulação utilizando um código computacional desenvolvido no aplicativo $\mathrm{R}$ apenas para esses dados. Além da reamostragem, o programa calculou os valores da estatística descritiva para o conjunto de dados obtidos (Tabela III). Esses resultados foram utilizados para realizar a inferência inicial dos valores amostrais medidos. A média aritmética e a mediana apresentaram-se iguais para todos os valores reamostrados de todas as minas (Tabela III). Além disso, os histogramas gerados para cada mina, a partir dos respectivos dados e plotados em conjunto com uma distribuição normal de mesma média e desvio-padrão, demonstraram uma possível adequação dos resultados com a distribuição normal, com exceção do conjunto de valores da Mina D que apresentou uma visível dispersão (Fig. 2). Apesar da adequabilidade à simetria de uma distribuição

Tabela III - Estatística descritiva da dose efetiva equivalente $\dot{\mathrm{H}}_{\mathbf{E}}\left(\mathrm{mSv} \cdot \mathrm{a}^{-1}\right)$ a partir de 100000 reamostras. [Table III - Descriptive statistics of the equivalent effective dose $\dot{H}_{E}\left(m S v \cdot a^{-1}\right)$ from 100000 samples.]

\begin{tabular}{cccccccc}
\hline & Mínimo & $\begin{array}{c}\text { Primeiro } \\
\text { quartil }\end{array}$ & Mediana & $\begin{array}{c}\text { Média } \\
\text { aritmética }\end{array}$ & $\begin{array}{c}\text { Terceiro } \\
\text { quartil }\end{array}$ & Máximo & $\begin{array}{c}\text { Intervalo de } \\
\text { confiança (95\%) }\end{array}$ \\
\hline Mina A & 1,37 & 1,45 & 1,47 & 1,47 & 1,49 & 1,55 & $1,42-1,52$ \\
Mina B & 1,44 & 1,53 & 1,55 & 1,55 & 1,56 & 1,64 & $1,51-1,59$ \\
Mina C & 1,63 & 1,92 & 1,98 & 1,98 & 2,04 & 2,30 & $1,80-2,15$ \\
Mina D & 1,71 & 2,02 & 2,15 & 2,15 & 2,28 & 2,95 & $1,78-2,52$ \\
\hline
\end{tabular}



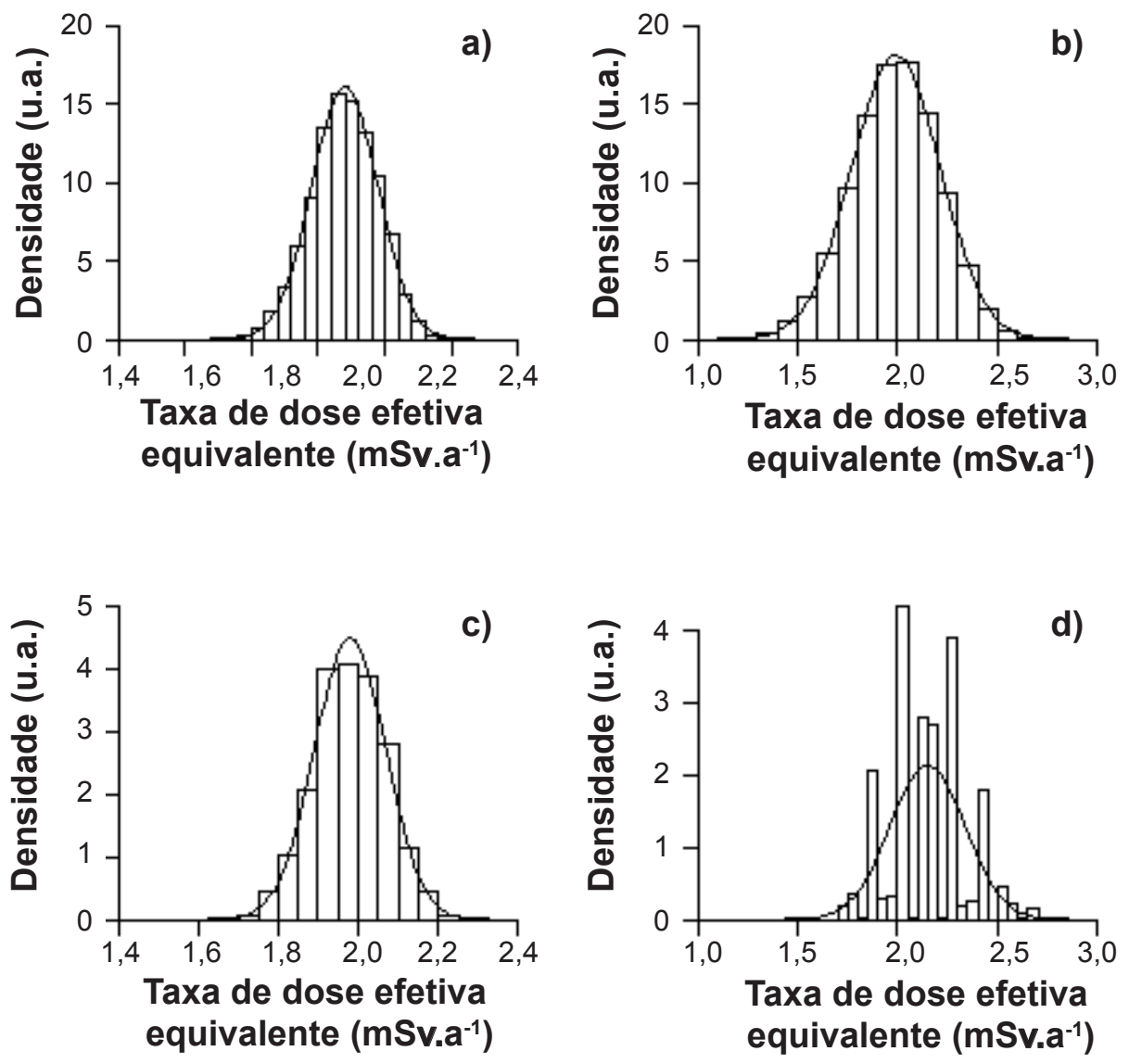

Figura 2: Histogramas das reamostras das taxas de dose efetivas equivalentes das minas estudadas: (a) mina A; (b) mina B; (c) mina C; e (d) mina D.

[Figure 2: Histograms of the resamples of the equivalent effective dose rates of the mines studied: (a) mine $A$; (b) mine $B$; (c) mine $C$; and (d) mine D.]

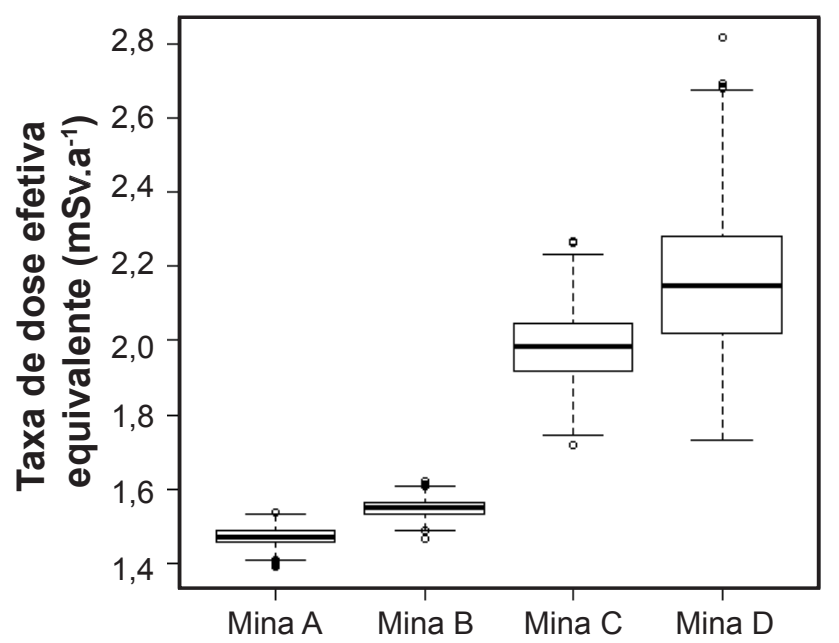

Figura 3: Gráficos de caixa (boxplot) das reamostras das taxas de dose efetivas equivalentes das minas estudadas.

[Figure 3: Boxplot of the resamples of the equivalent effective dose rates of the mines studied.]

normal, os gráficos das minas $\mathrm{A}, \mathrm{B}$ e $\mathrm{C}$ apresentaram também caudas alongadas à direita e à esquerda dos gráficos, indicando uma quantidade razoável de valores discrepantes

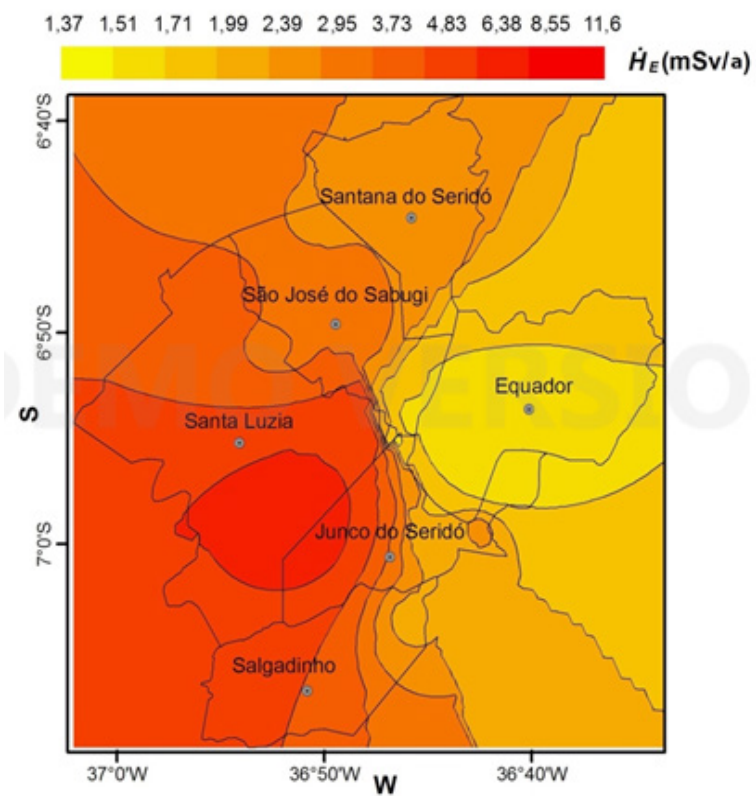

Figura 4: Carta de isodoses das amostras das taxas de dose efetivas equivalentes das minas estudadas.

[Figure 4: Isodoses charts of the samples of the equivalent effective dose rates of the mines studied.] 
menores e maiores que a medida de tendência central.

Em termos científicos é possível afirmar que a região no entorno da mina D apresentou valores de dose efetiva equivalente levemente mais acentuados quando comparados com as outras minas e com o valor médio divulgado pela UNSCEAR, além de apresentar uma maior dispersão dos dados e um razoável número de valores discrepantes em relação à média aritmética. As extensões das caudas das distribuições das minas $\mathrm{A}, \mathrm{B}$ e $\mathrm{C}$ corroboram inclusive a existência desses pontos discrepantes, indicando a possível existência de anomalias radiológicas pontuais em solos e rochas da região. É possível visualizar também esses pontos discrepantes além dos valores máximos obtidos através dos gráficos de caixa produzidos a partir dos valores reamostrados. A análise da dispersão pode ser visualizada através dos gráficos de caixa (boxplot) das 100000 reamostras das medições obtidas em cada mina. É possível comparar a dispersão interna de cada reamostra, como também entre minas (Fig. 3).

$O$ perfil radiométrico da PPBS pode ser visualizado através do mapa de curvas de isodose. Normalmente, esse é um recurso utilizado para avaliar a distribuição e intensidade da dose de fontes artificiais de radiação ionizante, principalmente de equipamentos médicos. Porém, passou a ser uma forma visual clara para as áreas monitoradas na radioecologia. A intensidade da taxa de dose efetiva equivalente nas minas localizadas nos municípios de Salgadinho e Junco do Seridó, ambos no estado da Paraíba, apresenta valores superiores em relação às outras minas (Fig. 4). As curvas de isodose demonstraram que ocorre uma maior intensidade da taxa de dose efetiva equivalente nas áreas mais próximas das minas identificadas como C e D, respectivamente nos municípios de Junco do Seridó e Salgadinho, concordando então com os valores da reamostragem Monte Carlo Bootstrap.

\section{CONCLUSÕES}

Inicialmente é importante afirmar que os valores de doses medidos no ar, no caso deste trabalho, não devem ser considerados quanto a estimativas de riscos determinísticos em relação a exposições diretas. Além deste fato, valores de doses estabelecidos por órgãos regulamentadores, como é o caso da UNSCEAR, apenas representam uma média global, mas não devem ser considerados como definitivos. É importante entendê-los como o limiar para a realização de análises e intervenções subsequentes, contribuindo para uma estimativa de possíveis riscos estocásticos associados aos valores obtidos nos campos de mineração. Não foi possível compor o quadro desejado com o máximo de medições no local do estudo. Porém, as medidas obtidas neste estudo foram utilizadas para estimar as taxas de doses efetivas equivalentes locais através de simulação matemática, contribuindo para o banco de dados nacional e servindo de base para a criação e aplicação de modelos matemáticos que definam as vias de transferência dos radionuclídeos naturais nessa região, favorecendo a identificação de possíveis riscos associados ao meio ambiente, podendo ser projetados para a população, além de contribuir para um melhor entendimento dos efeitos decorrentes da radioatividade natural e sua correlação com os aspectos ambientais e sociais. Apenas observando os valores da estatística descritiva foi possível concluir, supondo uma representatividade dos valores amostrais em relação à população estudada, uma pequena variação dos valores de taxa de dose efetiva equivalente em relação ao valor médio de 2,4 mSv. $\mathrm{a}^{-1}$ divulgado pela UNSCEAR. É uma inferência estatística importante que demonstra uma baixa exposição à radiação ionizante decorrente dos radioisótopos naturais. Porém, é importante destacar o problema do acesso às minas, portanto as medições realizadas geraram um número reduzido de dados e provenientes de regiões mais afastadas do problema analisado. Outra observação importante é a pesquisa por valores de dose efetiva equivalente no ar, a um metro de distância do solo, basicamente procurando por radiação gama proveniente do subsolo devido à produção de Ra-226, e de seus dependentes, a partir das séries do U-238 e do Th-232. O grupo RAE, diante dos resultados obtidos e das dificuldades enfrentadas neste projeto, já providenciou uma nova expedição para obtenção de amostras de solo, rocha e água para execução de uma dosimetria com maior acurácia através do método de espectroscopia de radiação gama utilizando o Laboratório de Análises Ambientais e Radioecológicas (LAAR) do DEN/UFPE. Esta pesquisa demonstrou também que em casos nos quais o número de valores amostrais for pequeno, é possível utilizar métodos de reamostragem, no caso Monte Carlo Bootstrap, para obtenção de pseudoamostras em um número suficiente para realização da inferência estatística do problema.

\section{AGRADECIMENTO}

Os autores agradecem ao DEN-UFPE por viabilizar os recursos financeiros, equipamentos e infraestrutura necessária para a realização desta pesquisa.

\section{REFERÊNCIAS}

[1] G. Viruthagiri, B. Rajamannan, K.S. Jawahar, Radiat. Prot. Dosim. 157, 3 (2013) 383.

[2] D.J. Brenner, R. Doll, D.T. Goodhead, E.J. Hall, C.E. Land, J.B. Little, J.H. Lubin, D.L. Preston, R.J. Preston, J.S. Puskin, E. Ron, R.K. Sachs, J.M. Samet, R.B. Setlow, M. Zaider, Proc. Natl. Acad. Sci. USA, 100, 24 (2003) 13761.

[3] J. Hála, J.D. Navatril, Radioactivity, ionizing radiation and nuclear energy, $2^{\text {nd }}$ Ed., Konvoj, Berkova, Czech Republic (2003) 190.

[4] Un. Nat. Sci. Com. Effects Atomic Radiation UNSCEAR, "Sources and effects of ionizing radiation", Report Vol. 1 (2008) 322.

[5] Min. Minas Energ. - MME, "Plano nacional de mineração 2030" (2010) 157.

[6] Inst. Pesq. Econ. Apl. - IPEA, "Resíduos sólidos da atividade de mineração", Coord. Min. Meio Ambiente (2012). 
[7] A.C. Silva, M. Vidal, M.G. Pereira, REM: Rev. Esc. Minas 54, 2 (2001) 133.

[8] E.R. Soeiro, "Caulim", Sumário Mineral, DNPM/PA (2014).

[9] F.A.N.G. Silva, "Estudos de caracterização tecnológica e beneficiamento do caulim da região Borborema-Seridó", Diss. Mestr., Univ. Fed. Rio Janeiro, Rio de Janeiro (2007) 7.

[10] J.R.A. Dantas, L.B. Barros, V.C. Souza, A.A.F. Mont'Alverne, "Distritos mineiros do Nordeste Oriental", Depto. Nac. Prod. Miner. - DNPM, Recife (2000) 61.

[11] Int. Atomic Energy Ag. - IAEA, "Exposure of the public from large deposits of mineral residues", IAEA, Vienna, Austria (2011) 1.

[12] N.N.M. Avelino, H.C. Santos, H.J. Dantas, S.C. Silva, C.A. Leite, I.A. Leite, in: Anais VII Congr. Norte Nordeste Pesq. Inov., Palmas, TO (2012).

[13] M.N.A. de Sousa, J.C. Alchieri, C\&D Rev. Eletrôn. Fainor 4, 1 (2011) 20.

[14] V.F.S. Pastura, T.F.C. Campos, R.A. Petta, in: Congr. Radioprot. Portug. Lang. Countr., Recife, PE (2011).

[15] V. Alves, E.R.R. Rochedo, N.M.P.D. Ferreira, in: IX Latin Am. IRPA Reg. Congr. Radiat. Prot. Saf., Rio de Janeiro, RJ (2013).

[16] Un. Nat. Sci. Com. Effects Atomic Radiation UNSCEAR, "Report to the General Assembly" 1 (2000) 68.

[17] M.A. Baloch, A.A. Qureshi, A. Waheed, M. Ali, M.N.
Ali, M. Tufail, S. Batool, M. Akram, O. Iftikhar, H.Q. Avyyum, S. Manzoor, H. Khan, J. Radiat. Res. 53 (2012) 41. [18] N.W. El-Dine, A. Soror, A. El-Shershaby, S.M. ElBashi, F. Ahmed, Appl. Radiat. Isot. 60, 1 (2004) 105.

[19] S. Turhan, I.H. Arikan, H. Demirel, N. Güngor, Radiat. Phys. Chem. 80, 5 (2011) 620.

[20] R. Ravisankar, K. Vanasundari, A. Chandrasekaran, A. Rajalakshmi, M. Suganya, P. Vijayagopal, V. Meenakshisundaram, Appl. Radiat. Isot. 70, 4 (2012) 699.

[21] B.M.A. Barbosa, "Estudo do potencial mineral para U-ETR com base na geoquímica e química mineral do Depósito Uranífero de São José de Espinharas (PB)", Diss. Mestr., Univ. Fed. Pernambuco, Recife (2012) 22.

[22] G.F. Knoll, Radiation detection and measurement, John Willey and Sons, New York (2000) 231.

[23] A.N.C. da Silva, "Análise estatística de dados radioecológicos discrepantes usando o método Monte Carlo Bootstrap", Diss. Mestr., Univ. Fed. Pernambuco, Recife (2013) 22.

[24] L.C. Matsushima, "Determinação das curvas de isodose e confirmação do planejamento em Radioterapia de Intensidade Modulada - IMRT convencional empregando as técnicas de termoluminescência, luminescência opticamente estimulada e detectores semicondutores", Tese Dr., Inst. Pesq. Energ. Nucl., Univ. S. Paulo, S. Paulo (2015) 60.

(Rec. 10/01/2017, Rev. 27/05/2017, 03/07/2017, Ac. 26/07/2017) 